\title{
Biología reproductiva de Brycon henni (Teleostei: Bryconidae) y estrategias de conservación para los ríos Nare y Guatapé, cuenca del río Magdalena, Colombia
}

\section{Néstor Javier Mancera-Rodríguez}

Universidad Nacional de Colombia, Sede Medellín. Departamento de Ciencias Forestales, Grupo de investigación Ecología y Conservación de Fauna Silvestre, Calle 59A No. 63-20, Bloque 20, oficina 211, Medellín, Colombia; njmancer@unal.edu.co

Recibido 04-XI-2016. Corregido 04-V-2017. Aceptado 06-VI-2017.

\begin{abstract}
Reproductive biology of Brycon henni (Teleostei: Bryconidae) and conservation strategies for the Nare and Guatapé rivers, Magdalena River Basin, Colombia. Fish can modify features of their life histories through evolutionary mechanisms to ensure the maintenance of their populations in response to environmental changes and/or fishing pressure. The reproductive biology of characid fish $B$. henni, in the Nare and Guatapé Rivers, Magdalena River Basin, Colombia was studied, and suggestions for conservation are presented. Ten field samplings were carried out between October 2007 and February 2012, in periods of rain, dry, transition from rain to dry and transition from dry to rain. A total of 848 specimens were collected, 360 of which were females, 441 males and 47 indeterminate. Males predominated in the catches, and the sex ratio of 1.00: 0.82 deviated significantly from the theoretical distribution 1:1. The average size captured for the total number of individuals studied was $121.5 \mathrm{~mm}$ SL $(38.5-304.0 \mathrm{~mm})$, with $133.1 \mathrm{~mm}$ SL for females (53.5-304.0), and $116.9 \mathrm{~mm}$ SL (38.5-232.5) for males. Based on the monthly variation of the gonadosomatic index (GSI) and the proportion of mature specimens, the spawning season occurs during periods of highest water levels in the rainy season. The lower values of hepatosomatic index HSI that coincide with the maximum values of the GSI, suggest that liver substances are transferred during the maturation of the ovary. The mean size at sexual maturity $\left(\mathrm{L}_{50}\right)$ of $B$. henni was $171.5 \mathrm{~mm} \mathrm{SL}$ in females, and $132.6 \mathrm{~mm} \mathrm{SL}$ in males. The fecundity varied from 655 to 8 213 oocytes/female (mean $=2072, \mathrm{SD}=1648)$ and relative fecundity was 16.1 oocytes $/ \mathrm{g}$ of total weight $(\mathrm{SD}=$ 5.9). The diameter of the oocytes was $1.24 \mathrm{~mm}(\mathrm{SD}=0.23)$. A similar development was observed in all oocytes, suggesting massive spawning activity concentrated in short periods of time. The protection of creeks and riparian habitats is a conservation priority, and protected areas such as fluvial nature reserves should be created to guarantee the survival of this species. Environmental education activities, along with water decontamination should be developed. For natural populations of $B$. henni is recommended to establish a minimum catch size of $270 \mathrm{~mm}$ SL (300 mm TL) combined with closed fishing seasons during periods of reproduction. Rev. Biol. Trop. 65 (3): 1105-1119. Epub 2017 September 01.
\end{abstract}

Key words: freshwater tropical fish, sexual maturity, Andes, fluvial nature reserves.

B. henni (Eigenmann 1913) es una especie con distribución restringida a la cuenca del Magdalena-Cauca y es de gran importancia como fuente proteínica en la alimentación de las poblaciones humanas ribereñas. La sabaleta $B$. henni se encuentra en sistemas lóticos y lénticos, con temperatura del agua entre 18 y 29 ${ }^{\circ} \mathrm{C}$, pero prefiere aguas claras y turbulentas, con sustratos duros compuestos de rocas y grava
(Builes \& Urán, 1974; Montoya-López, Carri1lo, \& Olivera, 2006a). Es altamente susceptible a cambios en las características físico-químicas del agua, en particular a la disminución del contenido de oxígeno disuelto (Builes \& Urán, 1974), así como a la contaminación del agua, lo que puede ocasionar disminuciones significativas en el número de individuos dentro de sus poblaciones, y la sobreexplotación podría 
conllevar a la pérdida de su variabilidad genética (Hurtado-Alarcón, Mancera-Rodríguez, \& Saldamando-Benjumea, 2011).

Algunos aspectos reproductivos de la especie han sido estudiados por Builes y Urán (1974) que determinaron las épocas de desove $\mathrm{y}$ describieron las gónadas y el comportamiento sexual de la especie en el río Nare y otros ríos de la cuenca del río Magdalena, y por Martínez-Orozco y Vásquez-Zapata (2001) que definieron los periodos de desove y la fecundidad relativa de la especie en el embalse de la Salvajina, al sur de Colombia en el Alto Cauca. Igualmente se ha estudiado la maduración gonadal en hembras y su relación con variables ambientales en cautiverio (Arboleda-Chacón, Olivera, Tabares, Echeverri, \& Serna, 2005), y se ha realizado la descripción anatómica e histológica de las gónadas (Montoya-López, Tabares, Echeverri, Arboleda, \& Olivera-Ángel 2006b).

La especie presenta dimorfismo sexual, los machos adultos presentan en los radios de su aleta anal espinas pequeñas (rugosas al tacto), mientras que las hembras solo las presentan en su época de madurez reproductiva (MontoyaLópez et al., 2006a; Lenis-Sucerquia, CruzCasallas, \& David-Ruales, 2015), y realiza migraciones laterales de mediano alcance entre los cauces principales de los ríos y arroyos o quebradas adyacentes, con fines reproductivos, o para la búsqueda de mejores condiciones ambientales (Builes \& Urán, 1974; Perdomo, 1978; Martínez-Orozco, \& Vásquez-Zapata, 2001; Arboleda-Chacón et al., 2005).

Los objetivos de este trabajo fueron determinar las principales características reproductivas de la sabaleta $B$. henni, en afluentes de los ríos Nare y Guatapé, cuenca del río Magdalena en Colombia, y proponer diferentes acciones para la conservación de la especie. Esto contribuirá a la formulación de programas de conservación de la especie que es de alta importancia alimentaria en las comunidades humanas en su área de distribución.

\section{MATERIALES Y MÉTODOS}

Localización y descripción del área de estudio: El trabajo se realizó en 12 estaciones ubicadas en afluentes de los ríos Nare y Guatapé en los municipios de San Vicente, Concepción, San Roque, Alejandría, San Rafael y San Carlos, departamento de Antioquia, en la cuenca del río Magdalena, Colombia (Cuadro 1). La zona tiene características de bosque húmedo tropical montano bajo (Holdridge, 1967), zonobioma húmedo tropical del valle del río Magdalena, con una temperatura promedio de $18{ }^{\circ} \mathrm{C}$ para San Vicente, Concepción y Alejandría, y de $23{ }^{\circ} \mathrm{C}$ para San Roque, San Rafael y San Carlos, y un patrón de precipitación bimodal con picos en los meses de mayo y octubre, con un promedio anual de $5720 \mathrm{~mm}$ de lluvia para la estación La Araña (2308067 Red Hidrometeorológica de Empresas Públicas de Medellín EE.PP.M) y de $4368 \mathrm{~mm}$ para la estación Embalse de Playas (2308799 - Red Hidrometeorológica de EE.PP.M). El territorio es montañoso, con topografía ondulada, y las quebradas drenan hacia la cuenca media del río Magdalena; éstas se caracterizan por ser torrenciales, de aguas muy claras y sin planicie aluvial (Mancera-Rodríguez, Castellanos-Barliza, \& Urrego-Ballestas, 2016).

Caracterización del hábitat: Se midieron algunas variables fisicoquímicas in situ: oxígeno disuelto $(\mathrm{mg} / \mathrm{L})$, temperatura superficial del agua $\left({ }^{\circ} \mathrm{C}\right)$ con medidor Hanna (HI9828/4-01) y $\mathrm{pH}$ y conductividad $(\mu \mathrm{S} / \mathrm{cm})$ con medidor Hanna (9 811-5). Ancho y profundidad fueron medidos con flexómetro, y una vara graduada en cm. El tipo de sustrato se clasificó de acuerdo con la escala propuesta por Wentworth (1922).

Obtención de las muestras: Se realizaron diez muestreos de campo entre octubre de 2007 y febrero de 2012, que incluyeron períodos de lluvia (octubre y noviembre 2007, mayo 2008 y noviembre 2011), períodos secos (enero 


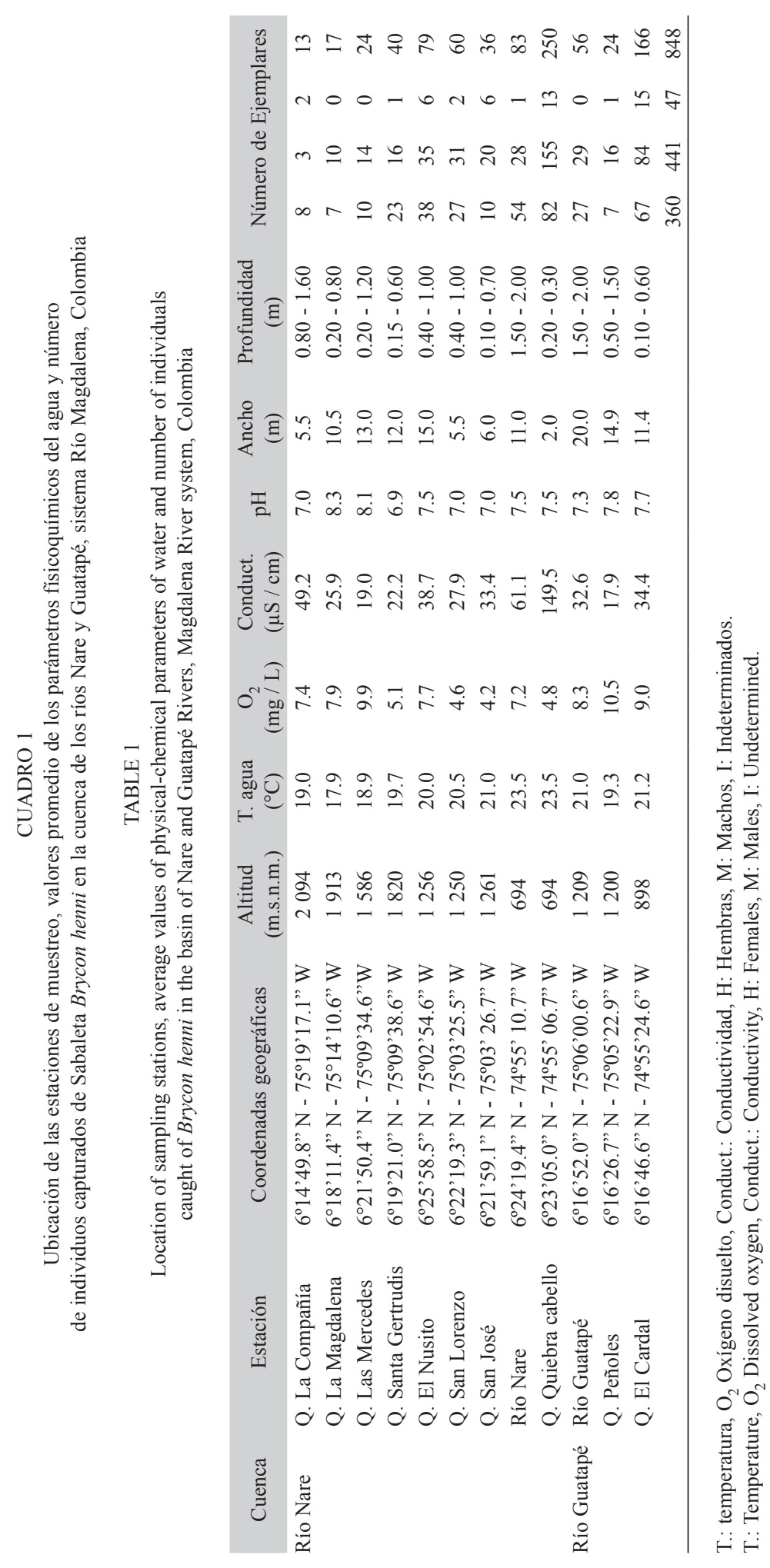


y marzo 2010, febrero 2012), transición de lluvia a seco (junio 2011) y transición de seco a lluvia (marzo 2008 y septiembre 2011). En cada punto, se realizaron capturas con un equipo de electropesca SAMUS 725MP, 30 lances de atarraya de $1 \mathrm{~cm}$ ojo de malla por estación y pesca con anzuelos.

Los ejemplares capturados se etiquetaron con fecha, lugar de captura y se trasladaron al laboratorio de Ecología y Conservación de Fauna Silvestre de la Universidad Nacional de Colombia, Sede Medellín, donde fueron lavados y preservados en alcohol al $70 \%$. Posteriormente, se midió su longitud estándar $(\mathrm{mm})$ con un calibrador digital hasta centésimas de milímetro, y se obtuvo su peso total (g) y eviscerado (g) con una balanza analítica (Ohaus Scout-Pro 202, 0.01 g). Los ejemplares se evisceraron uroventralmente para la extracción de las gónadas, las cuales fueron pesadas (g), fijadas en formol al $5 \% \mathrm{y}$ depositadas en frascos rotulados con la información del ejemplar. Ejemplares de $B$. henni se preservaron y están depositados en los Laboratorios de Ictiología de la Universidad del Quindío, Armenia, Colombia (IUQ) y en la colección de peces de la Universidad Católica de Oriente, Colombia (CP-UCO).

La estimación de la relación longitud estándar (LE)-peso total (PT) se realizó de acuerdo a la metodología aplicada por Mancera-Rodríguez et al. (2016). Para contrastar diferencias de longitud estándar y peso total entre sexos, debido a que los datos no cumplieron con los supuestos de normalidad (prueba de Shapiro-Wilk) y homocedasticidad (prueba de Levene), se utilizó la prueba no paramétrica de Kruskall-Wallis y las comparaciones pareadas con la prueba Mann-Whitney con el programa estadístico PAST (Hammer, Harper, \& Ryan, 2001).

La proporción de sexos (machos: hembras) se estableció para cada período de muestreo y para cada clase de longitud, y se utilizó una prueba de Chi-cuadrado $\left(\chi^{2}\right)$ para determinar diferencias significativas. La distribución de tallas se estimó en intervalos de $20 \mathrm{~mm}$ de longitud estándar, de acuerdo a la ecuación: $\mathrm{A}=\mathrm{N} / \mathrm{R}$,
$\mathrm{N}=$ Max - Min y $\mathrm{R}=1+3.322 \log (\mathrm{n})$; donde $\mathrm{A}=$ amplitud de los intervalos, $\mathrm{n}=$ número de observaciones, $\mathrm{Max}=$ valor máximo, $\mathrm{Min}=$ valor mínimo.

Se determinó el sexo y el estadio de desarrollo gonadal de cada individuo mediante observación de las gónadas, y se siguió la escala de maduración propuesta por Holden y Raitt (1975): I: Inmaduro, II: Reposo, III: En maduración, IV: Maduro y V: Desovado (hembras) o espermiado (machos). La actividad reproductiva se evaluó mediante los cambios en la condición gonadal a través del tiempo, para lo que se calculó el índice gonadosomático (IGS) (Vazzoler, 1996) de la siguiente manera $\mathrm{IGS}=(\mathrm{Pg} / \mathrm{Pt}) \times 100$ donde, $\mathrm{Pg}$ es el peso de la gónada y Pt es el peso total del pez en gramos. Se calculó el factor de condición (K) según Anderson y Gutreuter (1983), el índice hepatosomático (IHS), la talla media de madurez sexual $\left(\mathrm{LE}_{50}\right)$ y la fecundidad absoluta de acuerdo a la metodología aplicada en Mancera-Rodríguez et al. (2016). Se estimó la relación entre la fecundidad absoluta (el número de ovocitos maduros por peso) y la longitud estándar, el peso total, el peso eviscerado y el peso de la gónada.

\section{RESULTADOS}

Características del hábitat: Los cuerpos hídricos estudiados presentaron sustratos pedregosos y/o arenosos-pedregosos, y presencia de rocas de gran tamaño dentro y en sus márgenes. Durante la época seca sus aguas fueron cristalinas y en época de lluvias turbias y con corriente. La temperatura superficial del agua presentó valores medios entre 17.9 y 23.5 ${ }^{\circ} \mathrm{C}$, el oxígeno disuelto entre 4.2 y $10.50 \mathrm{mg}$ / L, la conductividad entre 17.9 y $149.5 \mu \mathrm{S}$ / $\mathrm{cm}$, y el pH entre 6.9 y 8.3 (Cuadro 1). Las quebradas presentaron una profundidad media entre $0.20-0.30 \mathrm{~m}$ la menos profunda y entre 0.80 - $1.60 \mathrm{~m}$ la de mayor profundidad, y los ríos Nare y Guatapé una profundidad media entre $1.50-2.00 \mathrm{~m}$. El ancho promedio varió entre $2 \mathrm{~m}$ para la quebrada Quiebra Cabello y $20 \mathrm{~m}$ para el río Guatapé (Cuadro 1). 
En total se capturaron 848 ejemplares de B. henni, de los cuales 360 fueron hembras, 441 machos, y 47 individuos indeterminados (Cuadro 1). Los individuos estudiados estuvieron en un rango entre 38.5 y $304 \mathrm{~mm}$ de longitud estándar (LE) y peso total (PT) entre 0.9 y $599.6 \mathrm{~g}$; las hembras presentaron un rango entre 53.5 y $304.0 \mathrm{~mm}$ de LE y PT entre 2.2 y $599.6 \mathrm{~g}$, y los machos un rango entre $38.5 \mathrm{y}$ $232.5 \mathrm{~mm}$ de LE y PT entre 0.9 y $246.2 \mathrm{~g}$ (Cuadro 2). Los peces capturados en el cauce principal de los ríos Nare y Guatapé presentaron una talla media de $158.8(\mathrm{DE}=30) \mathrm{mm}$ de LE para las hembras, y de $156.2(\mathrm{DE}=32) \mathrm{mm}$ de LE para los machos; Por su parte, los peces capturados en las quebradas, presentaron una talla de 125.6 $(\mathrm{DE}=47) \mathrm{mm}$ de LE para las hembras y de $111.0(\mathrm{DE}=35) \mathrm{mm}$ de LE para los machos. Las diferencias de LE y de PT entre sexos fueron significativas (Mann-Whitney, $\mathrm{P}<0.001$ ), siendo las hembras más grandes y con mayor peso. El tipo de crecimiento, determinado por la prueba $\mathrm{t}$ de Student, mostró para $B$. henni un crecimiento alométrico positivo $(\mathrm{b}>3)$ con constantes alométricas (b) de 3.07 para hembras y de 3.10 para machos (Cuadro 2).

Proporción de sexos: Fue de 1.00: 0.82 (441 machos y 360 hembras), se desvió significativamente de la hipotética distribución esperada de $1: 1\left(X^{2}=8.19, \mathrm{P}=0.004\right)$. De acuerdo con la distribución de los sexos con relación a la longitud estándar, las hembras fueron significativamente más abundantes en tamaños entre $130.0 \mathrm{~mm}$ y $189.9 \mathrm{~mm}$, y mayores a $210 \mathrm{~mm}$ de LE. Por encima de los $250 \mathrm{~mm}$ de LE, todos los individuos capturados fueron hembras. Los machos fueron más frecuentes en tallas menores a los $129.9 \mathrm{~mm}$ de $\operatorname{LE}\left(X^{2}=47.90, \mathrm{P}<0.001\right.$, $\mathrm{gl}=13)$ (Fig. 1).

Índice gonadosomático: Los valores medios del índice gonadosomático (IGS) presentaron variaciones en cada muestreo, presentando un comportamiento similar en ambos sexos. Los altos registros de IGS se presentaron durante los períodos de mayor nivel de las aguas en período de lluvia en noviembre 2007 (Promedio IGS $=2.26$ y 4.25 para hembras y machos, respectivamente) y en período de transición de temporada de lluvia a sequía en junio 2011 (Promedio IGS= 2.04 y 3.06 para hembras y machos respectivamente) (Fig. 2). La escala de madurez sexual indicó clara relación con los rangos de IGS para ambos sexos. Los valores promedio más altos del IGS correspondieron al estadio IV de individuos maduros, con un valor promedio de IGS de 4.45 y 4.11 (hembras y machos respectivamente) (Cuadro 3). Los individuos en este estadio IV de maduración presentaron una talla entre los 75.7 y $304.0 \mathrm{~mm}$ de LE para hembras y entre los 82.7

CUADRO 2

Relación longitud estándar - peso total de la Sabaleta Brycon henni en la cuenca de los ríos Nare y Guatapé, sistema Río Magdalena, Colombia

TABLE 2

Length standard - Weight (LS -W) relationships for of Brycon henni in the basin of Nare and Guatapé Rivers, Magdalena River system, Colombia

\begin{tabular}{lcccccccccccc} 
& \multicolumn{3}{c}{ Longitud estándar $(\mathrm{mm})$} & \multicolumn{4}{c}{ Peso total $(\mathrm{g})$} & \multicolumn{4}{c}{ Confidence interval for b $(95 \%)$} \\
\multicolumn{1}{c}{ Sexo } & $\mathrm{N}$ & $\mathrm{X}$ & Min. & Max. & $\mathrm{X}$ & Min. & Max. & $\mathrm{a}$ & $\mathrm{b}$ & Min. & $\mathrm{Max}$ & $\mathrm{R}^{2}$ \\
Hembras & 360 & 133.1 & 53.5 & 304.0 & 61.4 & 2.2 & 599.6 & 0.000013 & 3.07 & 3.02 & 3.13 & 0.985 \\
Machos & 441 & 116.9 & 38.5 & 232.5 & 39.5 & 0.9 & 246.2 & 0.000011 & 3.10 & 3.04 & 3.16 & 0.982 \\
Total & 848 & 121.5 & 38.5 & 304.0 & 47.4 & 0.9 & 599.6 & 0.000012 & 3.08 & 3.04 & 3.16 & 0.985 \\
\hline
\end{tabular}

$\mathrm{N}$ : número de individuos; X: promedio; Min: mínimo; Max: máximo; $\mathrm{R}^{2}$ : coeficiente de determinación; a y b: parámetros de relación.

$\mathrm{N}$ : number of individuals; X: average; Min: minimum; Max: maximum; $\mathrm{R}^{2}$ : coefficient of determination; $\mathrm{a}$ and $\mathrm{b}$ : relationship parameters. 


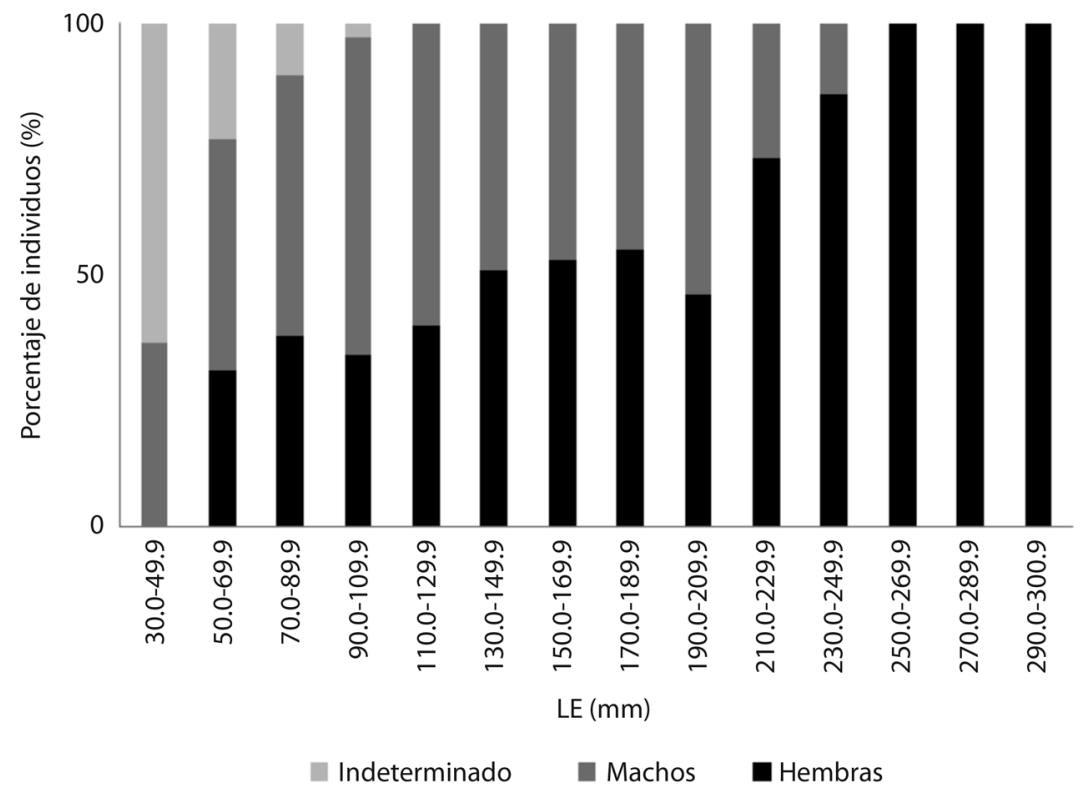

Fig. 1. Frecuencia de indeterminados, hembras y machos de B. henni en la cuenca de los ríos Nare y Guatapé, sistema Río Magdalena, Colombia, según el tamaño $(\mathrm{N}=848)$. LE = longitud estándar.

Fig. 1. Frequency of indeterminate, females and males of $B$. henni in the basin of Nare and Guatapé Rivers, Magdalena River system, Colombia, according to size $(\mathrm{N}=848)$. LE $=$ Standard length.

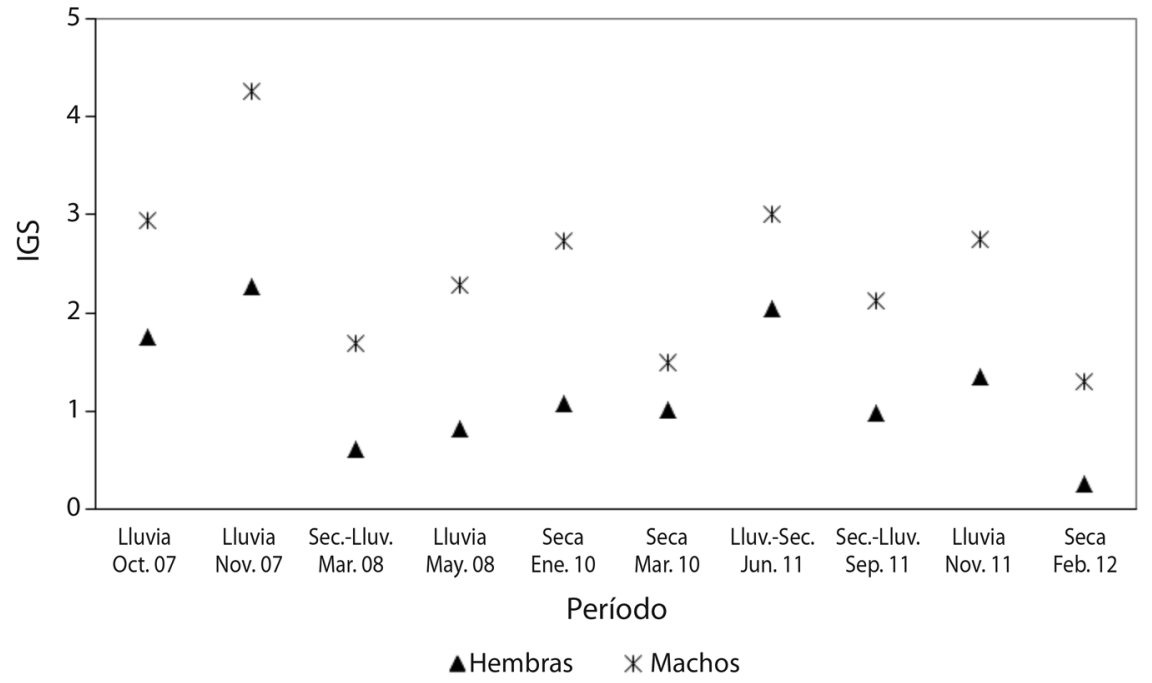

Fig. 2. Variación del Índice gonadosomático (IGS), valores medios (+/-SD) para ambos sexos de B. henni en la cuenca de los ríos Nare y Guatapé, sistema Río Magdalena, Colombia.

Fig. 2. Variation of the Gonadosomatic Index (GSI), the mean values (+/-SD) for both sexes of of $B$. henni in the basin of Nare and Guatapé Rivers, Magdalena River system, Colombia. 
CUADRO 3

Escala de madurez sexual con relación al valor medio del índice gonadosomático (IGS)

y las características macroscópicas de cada estadio en el desarrollo de ovarios y testículos de Brycon henni

TABLE 3

Sexual maturity scale with relation to the average value of the GSI, and the macroscopic characteristics of each stage in the development of Brycon henni ovaries and testes

\begin{tabular}{|c|c|c|c|c|}
\hline Sexo & Estadio & Descripción macroscópica & $\mathrm{n}$ & IGS \\
\hline \multirow[t]{5}{*}{ Hembras } & I & Ovarios pequeños y acintados sin productos sexuales, de color crema. & 84 & $0.209 \pm 0.15$ \\
\hline & II & $\begin{array}{l}\text { Ovarios rosados, translúcidos con vasos sanguíneos. Ovocitos invisibles a } \\
\text { simple vista. }\end{array}$ & 174 & $0.373 \pm 0.31$ \\
\hline & III & $\begin{array}{l}\text { Ovarios de mayor tamaño, de color crema con ovocitos de diferentes } \\
\text { tamaños a simple vista. }\end{array}$ & 31 & $0.726 \pm 0.39$ \\
\hline & IV & $\begin{array}{l}\text { Ovarios de gran tamaño y forma cilíndrica que ocupan gran parte de la } \\
\text { cavidad abdominal, de color amarillo y con gran irrigación sanguínea. } \\
\text { Ovocitos de tamaño uniforme a simple vista. }\end{array}$ & 52 & $4.449 \pm 2.38$ \\
\hline & V & $\begin{array}{l}\text { Ovarios casi vacíos, flácidos, de color rosado. Pueden ser visibles ovocitos } \\
\text { en el interior }\end{array}$ & 16 & $0.403 \pm 0.15$ \\
\hline \multirow[t]{5}{*}{ Machos } & I & Testículos pequeños, blancos y de forma plana o filamentosa. & 145 & $0.118 \pm 0.11$ \\
\hline & II & $\begin{array}{l}\text { Los testículos y conductos de esperma aumentan en tamaño, aparecen } \\
\text { aplanados de color blanco }\end{array}$ & 77 & $0.930 \pm 0.59$ \\
\hline & III & $\begin{array}{l}\text { Los testículos se ensanchan y aumentan de tamaño, presentan un color } \\
\text { blanco o crema. }\end{array}$ & 55 & $1.851 \pm 0.47$ \\
\hline & IV & $\begin{array}{l}\text { Testículos de gran tamaño y generalmente llenos de esperma de color } \\
\text { blanco. Ocupan gran parte de la cavidad abdominal y son muy irrigados. }\end{array}$ & 129 & $4.105 \pm 1.63$ \\
\hline & $\mathrm{V}$ & Testículos flácidos, traslúcidos y con residuos de esperma. & 35 & $0.217 \pm 0.17$ \\
\hline
\end{tabular}

(I: Inmaduro, II: Reposo, III: En maduración, IV: Maduro o puesta y V: Desovado (hembras), espermiado (machos). n: número de individuos.

(I: Immature, II: Rest, III: In maturation, IV: Mature or laying and V: Spawned (females), spermatozoon (males)

$\mathrm{n}$ : number of individuals.

y $232.5 \mathrm{~mm}$ de LE para los machos. Los peces presentaron mayor proporción de estadios de madurez sexual en fase IV (Maduro) y V (Desovado en hembras o espermiado en machos) durante los períodos de lluvia, tanto para hembras como para machos. Durante los períodos secos se observó mayor proporción de estadios de madurez sexual en fases I (Inmaduro), II (reposo) y III (en maduración) (Fig. 3).

Factor de condición (K) e Índice hepatosomático: El factor de condición $(\mathrm{K})$ tuvo los valores más altos durante los meses en que se presentaron los mayores valores del índice gonasomático (IGS) en los períodos de lluvia en octubre 2007 ( $\mathrm{K}=1.10$ y 1.18 para hembras y machos, respectivamente) y noviembre $2007(\mathrm{~K}=1.04$ y 1.10 para hembras y machos, respectivamente) y de transición de temporada lluvia a seca en junio $2011(\mathrm{~K}=1.09$ y 1.15 para hembras y machos, respectivamente); los menores valores se presentaron en el período de transición de temporada seca a lluvia, coincidiendo con menores valores de IGS (Fig. 4A). El IHS se registró contrario a $\mathrm{K}$, siendo mayor en el período seco de enero 2010 y en el período de transición de temporada seca a lluvia en septiembre 2011 cuando los peces presentaron menor desarrollo gonadal y menor valor de IGS (Fig. 4B). 

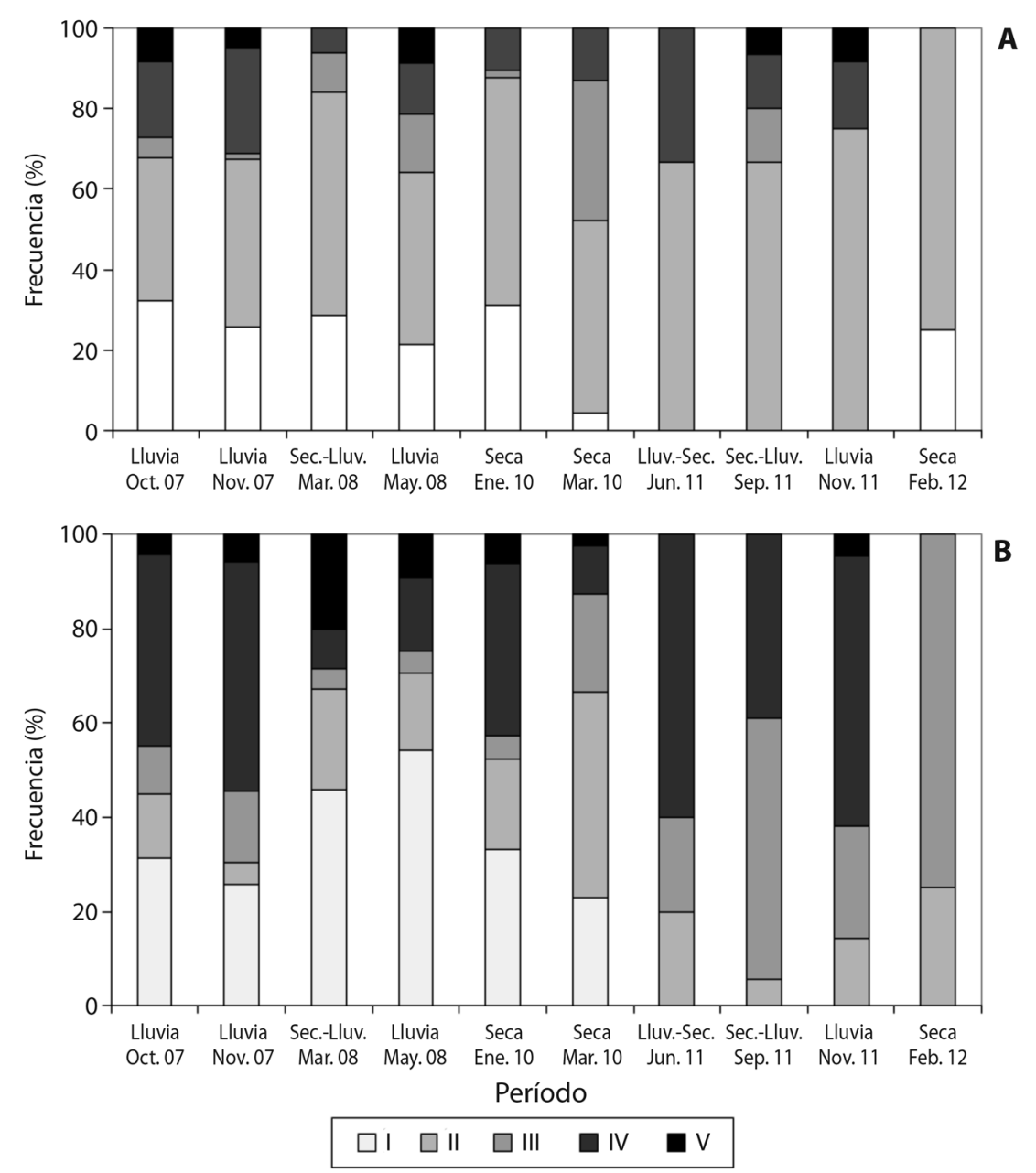

Fig. 3. Proporción de estadios de madurez sexual por período de hembras (A) y machos (B) de B. henni en la cuenca de los ríos Nare y Guatapé, sistema Río Magdalena, Colombia.

Fig. 3. Proportion of sexual maturity stages for each period of females (A) and males (B) of B. henni in the basin of Nare and Guatapé Rivers, Magdalena River system, Colombia.

Talla de madurez sexual: Los ejemplares maduros más pequeños se presentaron a una talla de 119.7 y $82.7 \mathrm{~mm}$ de LE para hembras y machos, respectivamente. La función logística mostró que la talla media de madurez sexual $\left(\mathrm{L}_{50}\right)$ fue de $171.5 \mathrm{~mm}$ de LE para las hembras y $132.6 \mathrm{~mm}$ para los machos (Fig. 5), y la talla de madurez sexual masiva $\left(\mathrm{L}_{95}\right)$ se alcanzó a los $223.3 \mathrm{~mm}$ de LE para las hembras y 200.5 $\mathrm{mm}$ para los machos.
Fecundidad: El número medio de ovocitos presentes en las gónadas de 42 hembras maduras de $B$. henni, en un rango de 119.7 hasta $279.1 \mathrm{~mm}$ de LE, capturadas durante el período reproductivo fue $2072(\mathrm{DE}=1648)$ con un rango entre 655 - 8213. El valor máximo de 8213 ovocitos se presentó a una talla de $279.1 \mathrm{~mm}$ LE. La fecundidad absoluta aumentó con la longitud estándar de acuerdo a la ecuación $\mathrm{F}=0.011 \mathrm{LE}^{2.31}\left(\mathrm{R}^{2}=0.63\right)$. La ecuación 

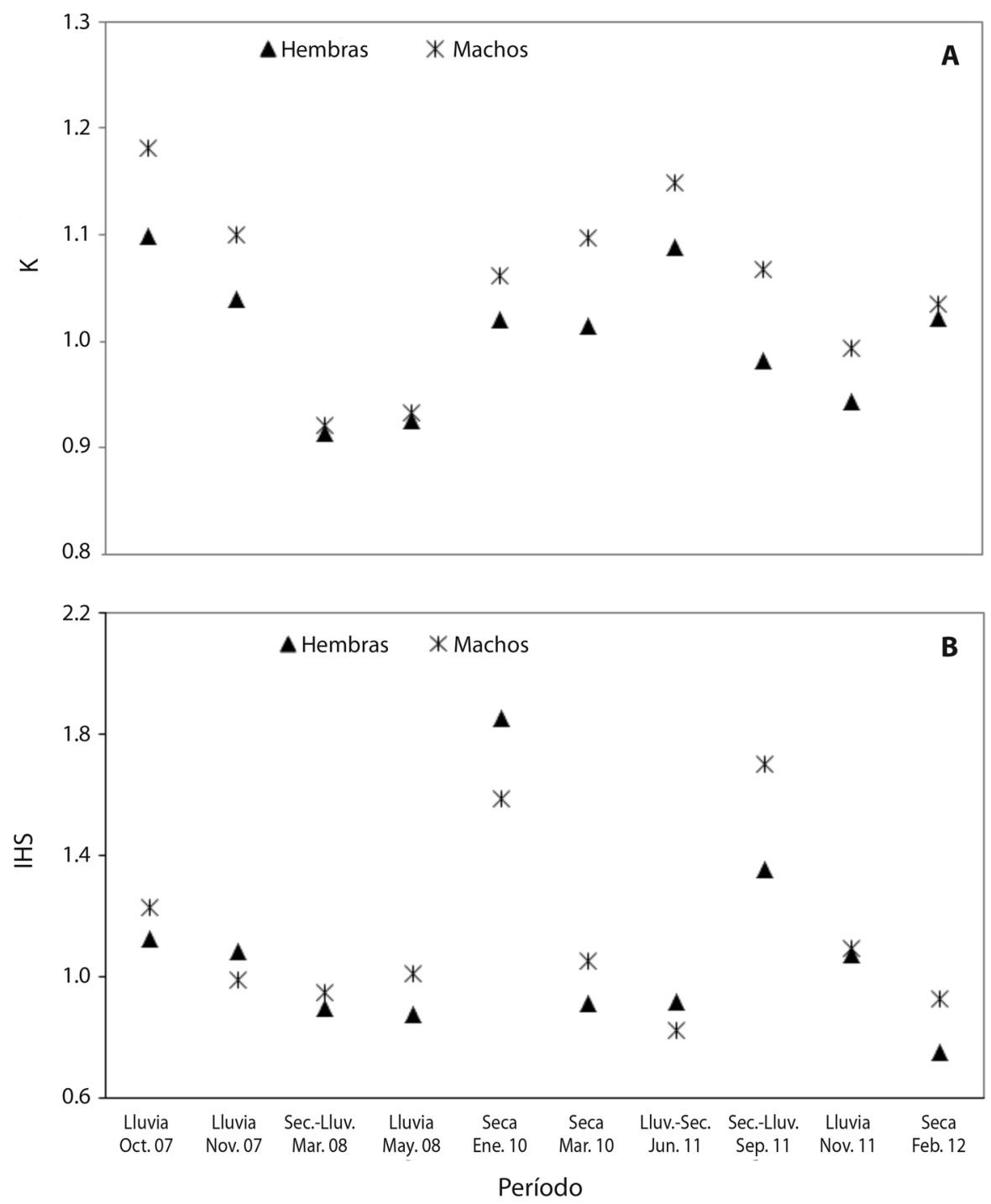

Fig. 4. Factor de condición (K) e índice hepatosomático (IHS) de hembras y machos de $B$. henni en la cuenca de los ríos Nare y Guatapé, sistema Río Magdalena, Colombia.

Fig. 4. Condition factor (K) and Hepatosomatic Index (HIS) by females and males of $B$. henni in the basin of Nare and Guatapé Rivers, Magdalena River system, Colombia.

que describe la relación entre la fecundidad absoluta $\mathrm{F}$ y peso eviscerado fue $\mathrm{F}=48.07$ $\mathrm{PEv}^{0.805}\left(\mathrm{R}^{2}=0.71\right)$ y con el peso de las gónadas $\mathrm{F}=751.1 \mathrm{Pg}^{0.614}\left(\mathrm{R}^{2}=0,67\right)$. La fecundidad relativa al peso total fue de 16.13 ovocitos / $\mathrm{g}$ $(\mathrm{DE}=5.9)$, al peso eviscerado de 21.55 ovocitos / g $(\mathrm{DE}=7.6)$, y al peso de las gónadas de 503.19 ovocitos / $\mathrm{g}(\mathrm{DE}=245.6)$.
El diámetro medio de los ovocitos varió entre 0.5 y $1.8 \mathrm{~mm}$ de diámetro, con un valor medio de $1.24 \mathrm{~mm}$ y un pico de la frecuencia máxima de diámetros entre 1.0 y $1.4 \mathrm{~mm}$. No hubo diferencia en el diámetro medio de los ovocitos presentes en las gónadas en relación con la longitud estándar (Correlación de Pearson $\mathrm{R}=-0.07 ; \mathrm{P}=0.66)$. Se observó 


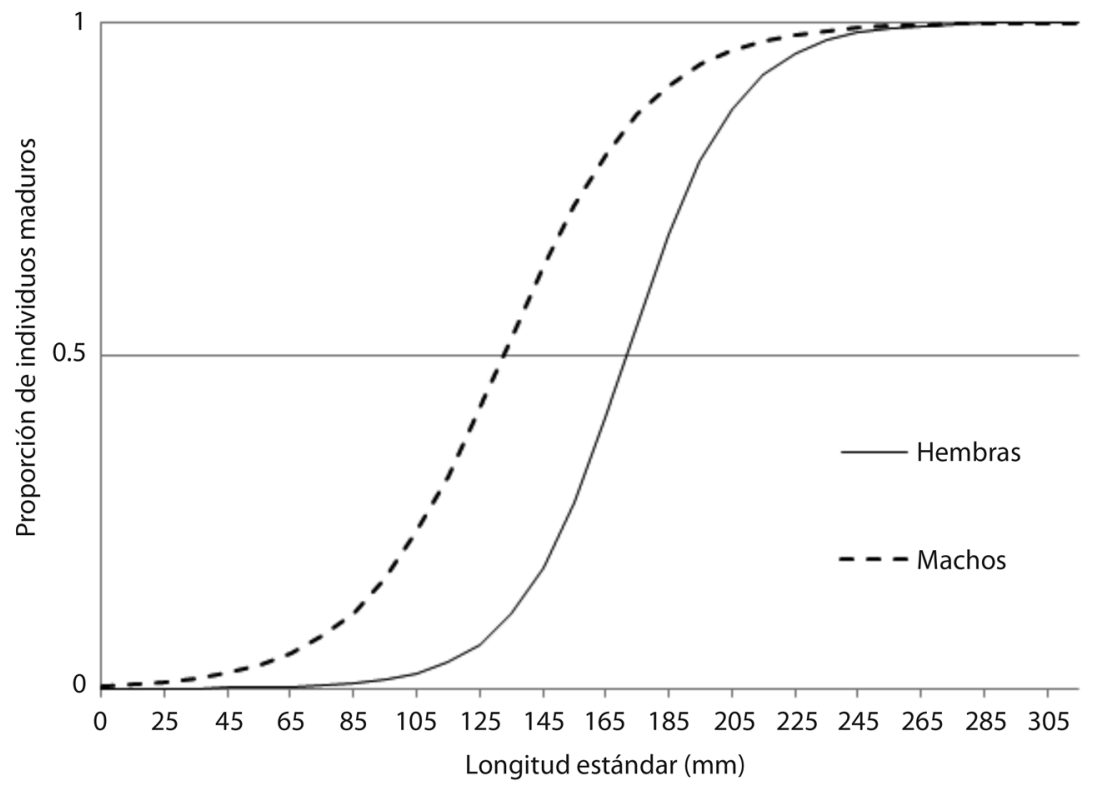

Fig. 5. Curva de madurez sexual y talla media de madurez sexual $\left(\mathrm{L}_{50}\right)$ para hembras y machos de $B$. henni en la cuenca de los ríos Nare y Guatapé, sistema Río Magdalena, Colombia.

Fig. 5. Curve of sexual maturity and the mean size of sexual maturity $\left(\mathrm{L}_{50}\right)$ for females and males of $B$. henni in the basin of Nare and Guatapé Rivers, Magdalena River system, Colombia.

un desarrollo similar en los ovocitos, lo que sugiere un desove masivo concentrado en cortos períodos de tiempo.

\section{DISCUSIÓN}

La proporción de sexos se presentó a favor de los machos (1.00: 0.82), y cambió con la longitud, siendo los machos más frecuentes en tallas menores a los $129.9 \mathrm{~mm}$ de LE, y las hembras entre $130.0 \mathrm{~mm}$ y $189.9 \mathrm{~mm}$, y por encima de los $240 \mathrm{~mm}$ de LE, donde todos los individuos capturados fueron hembras. Para $B$. henni, Beltrán (1978) reportó una relación de 1: 1.17 en el embalse Troneras (Antioquia), y Martínez-Orozco y Vásquez-Zapata (2001) de 1: 1.84 , favorables a las hembras.

La especie registró un crecimiento alométrico positivo que indicó una ganancia en peso mayor en relación con la longitud estándar. Lo cual es contrario a lo reportado para la especie por Zuluaga-Gómez, Giarrizzo, Andrade, y Arango-Rojas (2014), quienes encontraron un crecimiento alométrico negativo $(b=2.83)$ en la cuenca media y baja del río Cauca. Los valores medios de LE y PT fueron significativamente diferentes entre sexos, con mayor longitud las hembras, lo que coincide con lo reportado para la especie por Beltrán (1978), MontoyaLópez et al. (2006a) y Restrepo-Escobar, Hurtado-Alarcón, Mancera-Rodríguez, y Márquez (2016). Igualmente, se evidenció una mayor talla en los ejemplares capturados en el cauce principal de los ríos Nare y Guatapé, con relación a las quebradas, lo que puede deberse a que los individuos juveniles permanecen en los tributarios, y luego migran al canal principal del río a continuar su crecimiento, y a que durante los períodos reproductivos, los ejemplares maduros ingresan nuevamente a las quebradas a desovar. Restrepo-Escobar et al. (2016), encontraron que los peces de las quebradas presentaron cuerpos significativamente más pequeños y más estrechos que los presentes en el canal principal de los ríos Nare y Guatapé, como resultado de posibles respuestas fenotípicas a la velocidad del agua y la profundidad del cuerpo hídrico. 
El período de mayor desarrollo gonadal de $B$. henni en las quebradas afluentes de los ríos Nare y Guatapé, se estableció cuando empezó a subir el nivel de las aguas, durante las lluvias, lo cual debe facilitar los movimientos migratorios entre los ríos y las quebradas para el desove. En este período, se observó un aumento del Índice Gonadosomático y el Factor de Condición, relacionados con un alto porcentaje de individuos en estadio de madurez sexual IV. Kerguelen-Durango y Atencio-García (2015), destacan que en sistemas acuáticos andinos, los patrones hidrológicos y el transporte de nutrientes generado por el período lluvioso, son definitivos para la selección del tiempo óptimo de reproducción, y Andrade y Braga (2005) afirman que la intensidad de la lluvia es el factor más importante en la sincronización de la reproducción de peces tropicales, por lo que el coincidir el período reproductivo con la estación de lluvias, puede ser una estrategia evolutiva para que los juveniles aprovechen la abundancia de recursos, ya que la productividad de la materia de origen alóctono es mayor durante este momento, al compararlo con la época seca (Winemiller, 1990).

Builes y Urán (1974) determinaron dos épocas de desove con picos máximos en abril y octubre, períodos de mayor precipitación, y Flórez (1999), Usma (2001) y Jiménez-Segura et al. (2016) reportaron que las épocas reproductivas de $B$. henni están asociadas con la estación lluviosa. Por el contrario, se reporta la existencia de una relación inversa entre la madurez y la pluviosidad y las épocas de desove con los máximos valores de temperatura (Perdomo, 1978; Arboleda-Chacón et al., 2005), o épocas periódicas de actividad reproductiva a lo largo de todo el año en embalses (Martínez-Orozco \& Vásquez-Zapata, 2001; Jiménez-Segura et al., 2016), ya que en éstos no hay un cambio drástico en el nivel del agua, como resultado de los patrones de lluvia, como ocurre en los sistemas acuáticos naturales, por lo que las especies de peces sólo tienen que encontrar las condiciones del hábitat favorables para el desove y reclutamiento (JiménezSegura et al., 2016).
El IHS se registró inverso al factor de condición $(\mathrm{K})$, fue menor en los períodos de mayor desarrollo gonadal de la especie y menor valor de IGS. Los valores de IHS en hembras normalmente descienden durante los estadios de maduración y maduros, debido a la transferencia de sustancias hepáticas durante la vitelogénesis (Selman \& Wallace, 1989). Los bajos valores durante los períodos de mayor madurez gonadal, permiten correlacionar la transferencia de sustancias hepáticas durante la maduración del ovario.

Los tamaños de primera maduración sexual se presentaron a una talla de 119.7 y $82.7 \mathrm{~mm}$ de LE para hembras y machos, respectivamente. Cerca de las tallas de ejemplares maduros más pequeños, reportadas por Montoya-López et al. (2006b), de $10.4 \mathrm{~cm}$ de LE., aunque más pequeña que la reportada por Builes y Urán (1974) de 160 y $150 \mathrm{~mm}$ de LT para hembras y machos, respectivamente. Se observó que los machos alcanzaron la madurez sexual a una talla menor que las hembras, similar a lo reportado para la especie por Magallanes (1989).

Se encontró una longitud máxima de 304 y $232.5 \mathrm{~mm}$ de LE para hembras y machos de $B$. henni, respectivamente. Este registro es menor al encontrado por Builes y Urán (1974), quienes reportaron en el río Nare una longitud máxima de $370 \mathrm{~mm}$, y $350 \mathrm{~mm}$ LT para hembras y machos, respectivamente, y por la reportada por Martínez-Orozco y VásquezZapata (2001) de $385 \mathrm{~mm}$ LT en el embalse La Salvajina (Departamento del Cauca).

La talla media de madurez sexual $\left(\mathrm{L}_{50}\right)$ fue de $171.5 \mathrm{~mm}$ de LE para las hembras y 132.6 $\mathrm{mm}$ LE para los machos. Este valor fue menor al reportado por Perdomo (1978) de $257.5 \mathrm{~mm}$ LT para hembras, y al reportado por MartínezOrozco y Vásquez-Zapata (2001) de $297 \mathrm{~mm}$ de LT y $279.5 \mathrm{~mm}$ LE para hembras y machos, respectivamente. Igualmente, Builes y Urán (1974), aunque no reportaron un valor de talla media de madurez sexual para ejemplares de $B$. henni colectados en el río Nare, si registraron que las tallas de comienzo de maduración fueron mucho mayores que las encontradas en el presente estudio. Las condiciones ambientales 
desfavorables pueden forzar a los individuos a tener una maduración temprana para reducir el riesgo de morir antes de reproducirse (Wootton, 1999). Factores como la fuerte presión de pesca sobre los individuos de mayor talla de la especie en la zona (megadesovadores), donde ha sido altamente capturada como parte de la subsistencia de los pobladores de la región, y por la pesca deportiva, pueden estar llevando a una selección direccional, que produce una reducción marcada del tamaño de maduración sexual de la especie. En este sentido, se conoce que la eliminación selectiva de los peces adultos de gran tamaño trae como resultado, que poblaciones explotadas maduren a edades más tempranas y tamaños más pequeños, invierten más energía en la reproducción, y alcanzan adultos de tamaño más pequeño por edad (Sharpe \& Hendry, 2009; Audzijonyte, Kuparinen, \& Fulton, 2013; Audzijonyte, Fulton, \& Kuparinen, 2015).

Las hembras más grandes produjeron más huevos por unidad de peso corporal y la fecundidad absoluta aumentó con la longitud estándar. Los valores de fecundidad absoluta de las hembras de B. henni (media de 2072 ovocitos) fueron menores a los reportados por Martínez-Orozco y Vásquez-Zapata (2001), quienes encontraron un valor medio de 11407 ovocitos, lo que se debe a que evaluaron peces con tallas mayores, entre 272 y $330 \mathrm{~mm}$ de LT. Sin embargo, la fecundidad relativa (16.13 ovocitos / g) fue similar a la reportada por estos autores de 15.85 ovocitos / g. El diámetro de los ovocitos varió entre 0.5 y $1.8 \mathrm{~mm}$ de diámetro y el diámetro medio fue de $1.24 \mathrm{~mm}$, similar a lo reportado por Beltrán, Beltrán, y Sierra (1978) con diámetros entre 0.9 y $1.9 \mathrm{~mm}$.

Para B. henni se observó en los ovocitos, estados de desarrollo similares que sugieren desoves masivos concentrados en cortos períodos de tiempo. Builes y Urán (1974) y Lenis-Sucerquia et al. (2015) reportaron que las hembras presentaron ovoposición por etapas y que una cohorte de ovocitos se desarrolló de forma sincrónica. De acuerdo a las estrategias reproductivas propuestas por Winemiller (1989), la especie presenta una estrategia estacional con reproducción estacional y cíclica, sincronizada con el comienzo de la temporada de lluvias, sin cuidado parental, con moderada fecundidad y migraciones de desove. Heino, Kaitala, Ranta, y Lindström (1997) señalaron que las especies en las que las poblaciones exhiben sincronía espacial en la reproducción, o responden a un solo evento que actúa a escala regional, como la precipitación, tienen una mayor probabilidad de extinción porque son altamente sensibles a cualquier perturbación que pueda afectar este proceso.

Por lo anterior, es indispensable emprender acciones de conservación de la especie, ya que la pesca sin control, la afectación de los cuerpos hídricos por actividades ganaderas y agrícolas, y los procesos de contaminación por aguas residuales de las comunidades humanas asentadas en estas cuencas han disminuido drásticamente sus tamaños poblacionales. Es prioritaria la evaluación de la calidad ecológica y la protección de las quebradas y sus áreas de bosque ribereño, así como la declaración de áreas protegidas a nivel local o regional basadas en la protección de sus cuencas hídricas, ya que son ecosistemas de importancia para el desove, crecimiento y desarrollo hacia etapas adultas, de ésta y otras especies de peces nativos. Así mismo, la introducción de especies exóticas en los embalses ubicados en la cuenca de los ríos Nare y Guatapé, como la Carpa Cyprinus carpio, la Perca Americana Micropterus salmoides, o las Tilapias Oreochromis spp. han generado impactos ambientales negativos, porque depredan las especies de peces nativas o compiten por diferentes recursos con ellas. Por lo tanto, las autoridades ambientales y pesqueras deben establecer normativas que impidan la introducción de nuevas especies exóticas y los repoblamientos de las especies existentes en la región independientemente de que tengan posibilidades comerciales o económicas y garantizar un control sobre sus poblaciones actuales.

Igualmente, la construcción de los embalses en las cuencas de los ríos Nare y Guatapé inciden en el estado de las poblaciones de $B$. henni, al generar aislamiento por distancia y separación de grupos genéticos 
(Hurtado-Alarcón et al., 2011); por lo que cualquier tipo de liberación o introducción de ejemplares producidos en cautiverio, debe evitar que se mezclen individuos con una constitución genética diferente a la de las poblaciones receptoras. Se recomienda en lugar de programas de repoblación, que son costosos, dispendiosos y poco efectivos en resultados de recuperación de las poblaciones naturales, que se implementen otras estrategias relacionadas con la protección de cuencas y microcuencas, tales como la protección de vegetación ribereña, la reforestación con especies propias de la zona, la disminución o no uso de agroquímicos, y el tratamiento de aguas residuales de los diferentes municipios.

Se deben llevar a cabo actividades de sensibilización ambiental con las comunidades humanas de la zona, que incluyan la importancia de la Sabaleta como recurso alimenticio de subsistencia, y dirigir programas de conservación a los pescadores artesanales para evitar la pesca de la especie con artes no adecuadas, como atarrayas, chinchorros, trampas, barbasco y dinamita en ríos y quebradas. Se sugiere establecer para las poblaciones naturales de $B$. henni una talla mínima de captura de $270 \mathrm{~mm}$ de LE (300 mm de LT), a la cual los ejemplares tienen un peso superior a los $380 \mathrm{~g}$ y que garantiza que éstos se hayan reproducido. Esto permitiría, que ejemplares de mayor tamaño y peso, que producen más cantidad de huevos, los cuales a su vez también son de mayor tamaño, participen de los eventos reproductivos y generen un importante incremento de nuevos individuos a las poblaciones. Lo anterior implica la actualización de la normatividad legal vigente sobre el manejo de la pesca de la especie, ya que aunque la Resolución N 670 de 1971 del Instituto Nacional de los Recursos Naturales Renovables y del Ambiente (INDERENA) prohíbe su pesca, almacenamiento y transporte con fines de comercialización, permite su pesca con fines deportivos con caña y anzuelo y no especifica tallas mínimas o máximas de captura. Esta acción puede combinarse con períodos de veda aplicados en los períodos de lluvias en los meses de abril-mayo y octubre-noviembre que coinciden con los tiempos de mayor actividad reproductiva de la especie.

Finalmente, se requiere que las acciones de conservación de la especie sean articuladas con los planes de acción desarrollados por las empresas encargadas del manejo de las cuencas hídricas que abastecen los embalses presentes en el oriente Antioqueño (ISAGEN E.S.P. y Empresas Públicas de Medellín - EEPPM). Dichos planes deben contemplar las políticas, planes y estrategias desarrolladas por la Autoridad Nacional de Acuicultura y Pesca AUNAP como entidad encargada del manejo sostenible del recurso pesquero en el país y del Ministerio de Ambiente y Desarrollo Sostenible MADS como máxima Autoridad Ambiental encargada de velar por los recursos biológicos y genéticos de Colombia.

\section{AGRADECIMIENTOS}

A la empresa ISAGEN S.A. E.S.P., a la Corporación Autónoma Regional de los Ríos Negro y Nare -CORNARE y a la Universidad Nacional de Colombia por la financiación del proyecto 20101009235 "Estudio de la biología, ecología y diversidad genética de las poblaciones naturales de sabaleta Brycon henni en las cuencas de los ríos Nare y Guatapé, Antioquia”.

\section{RESUMEN}

Los peces pueden modificar características de sus historias de vida a través de mecanismos evolutivos para asegurar el mantenimiento de sus poblaciones en respuesta a los cambios ambientales y/o la presión de la pesca. Se estudió la biología reproductiva de $B$. henni en los ríos Nare y Guatapé, sistema Río Magdalena, Colombia, y se presentan sugerencias para la conservación de la especie. Se realizaron diez muestreos de campo entre octubre 2007 y febrero 2012, en períodos de lluvia, sequía, transición de lluvia a sequía y transición de seco a lluvia. Se recolectaron 848 especímenes, 360 hembras, 441 machos y 47 indeterminados. Los machos predominaron en las capturas, con una proporción de sexos de 1.00: 0.82 se desvió significativamente de la distribución teórica 1 : 1. La talla media de captura para el total de individuos estudiados fue de $121.5 \mathrm{~mm}$ de LE (38.5-304.0 mm), siendo para las hembras de 133.1 (53.5-304.0) $\mathrm{mm}$ de LE y para los machos de $116.9 \mathrm{~mm}$ de LE (38.5-232.5). Con base en la evolución mensual del índice gonadosomático 
(IGS) y la proporción de individuos maduros, la temporada de desove se produce durante los períodos de incremento del nivel del agua durante la temporada de lluvias. Los menores valores de IHS que coinciden con los valores máximos del IGS sugieren la transferencia de sustancias hepáticas durante la maduración del ovario. La talla media de madurez sexual $\left(\mathrm{L}_{50}\right)$ para los individuos de B. henni se alcanzó en las hembras a los $171.5 \mathrm{~mm}$ de LE y en los machos a los $132.6 \mathrm{~mm}$ de LE. La fecundidad fluctuó entre 655 y 8213 ovocitos/hembra (media= $2072 ; \mathrm{DE}=1648)$ y la fecundidad relativa fue de 16.13 ovocitos / $\mathrm{g}$ de peso total $(\mathrm{DS}=5.9)$. El diámetro de los oocitos fue de $1.24 \mathrm{~mm}$ $(\mathrm{SD}=0.23)$. Se observó un desarrollo similar en los ovocitos, lo que sugiere un desove masivo concentrado en cortos períodos de tiempo. La protección de quebradas y hábitats ribereños es prioritaria, y se deben crear áreas protegidas como las reservas naturales fluviales para garantizar la supervivencia de esta especie. Deben desarrollarse actividades de educación ambiental y descontaminación hídrica. Para las poblaciones naturales de $B$. henni se recomienda establecer una talla mínima de captura de $270 \mathrm{~mm}$ de LE (300 mm de LT), combinada con períodos de veda durante los períodos de reproducción de la especie.

Palabras clave: pez tropical de aguadulce, madurez sexual, Andes, reservas naturales fluviales.

\section{REFERENCIAS}

Anderson, R. O., \& Gutreuter, S. J. (1983). Length, weight, and associated structural indices. In L. A. Nielsen \& D. L. Johnson (Eds.), Fisheries Techniques (pp. 283-300). Maryland: American Fisheries Society, Bethesda.

Andrade, P., \& Braga, F. (2005) Reproductive seasonality of fishes from a lotic stretch of the Grande River, high Paraná River basin, Brazil. Brazilian Journal of Biology, 65, 387-394.

Arboleda, L., Olivera-Ángel, M., Tabares, C. J., Echeverri, A., \& Serna, D. F. (2005). Maduración gonadal en hembras de sabaleta (Brycon henni) y su relación con variables medioambientales. Politécnica, 1, 95-103.

Audzijonyte, A., Kuparinen, A., \& Fulton, E. A. (2013). How fast is fisheries-induced evolution? Quantitative analysis of modelling and empirical studies. Evolutionary Applications, 6, 585-595.

Audzijonyte, A, Fulton, E., \& Kuparinen, A. (2015). The impacts of fish body size changes on stock recovery: a case study using an Australian marine ecosystem model. ICES Journal of Marine Science, 72, 782-792.

Beltrán, C. N., Beltrán, I. C., \& Sierra, R. (1978). Notas preliminares sobre la reproducción y cultivo de la sabaleta (Brycon henni, Eigenman 1913). Bogotá, Colombia: Instituto de Recursos Naturales.
Beltrán, I. C. (1978). Aporte al estudio biológico pesquero del embalse Troneras (Antioquia) y alternativas para su manejo. Medellín, Colombia: Inderena, Regional Occidente.

Builes, J., \& Urán, A. (1974). Estudio del ciclo sexual de la Sabaleta Brycon henni Eigenmann. Su comportamiento y fecundación artificial. Actualidades Biológicas, 7(3), 2-12.

Flórez, P. E. (1999). Estudio biológico-pesquero preliminar de tres especies ícticas del Alto Río Cauca Embalse de Salvajina. Cespedesia, 23(73-74), 47-60.

Hammer, Ø., Harper, D. A. T., \& Ryan, P. D. (2001). PAST: Paleontological Statistics Software Package for Education and Data Analysis. Retrived from http:// palaeoelectronica. org/2001_1/past/issue1_01.htm

Heino, M., Kaitala V., Ranta E., \& Lindström, J. (1997) Synchronous dynamics and rates of extinction in spatially structured populations. Proceedings of the Royal Society of London. Series B: Biological Sciences, 264, 481-486.

Holden, M. J., \& Raitt, D. F. S. (1975). Manual de ciencia pesquera. Parte II. Métodos para investigar los recursos y su aplicación (Documento técnico 115). Roma, Italia: FAO.

Holdridge, L. R. (1967). Life zone ecology. San José, Costa Rica: Centro Científico Tropical.

Hurtado-Alarcón, J. C., Mancera-Rodríguez, N. J., \& Saldamando-Benjumea, C. I. (2011). Variabilidad genética en Brycon henni (Characiformes: Characidae) en la cuenca media de los ríos Nare y Guatapé, sistema Río Magdalena, Colombia. Revista de Biología Tropical, 59(1), 269-282.

Jiménez-Segura, L. F., Galvis-Vergara, G., Cala, P., GarcíaAlzate, C. A., López-Casas, S., Ríos-Pulgarín, M. I., ... Álvarez-León, R. (2016). Freshwater fish faunas, habitats and conservation challenges in the Caribbean river basins of north-western South America. Journal of Fish Biology, 89, 65-101.

Kerguelen-Durango, E., \& Atencio-García, V. (2015). Environmental characterization of the reproductive season of migratory fish of the Sinú River (Córdoba, Colombia). Revista MVZ Córdoba, 20, 4766-4778.

Lenis-Sucerquia, G. A., Cruz Casallas, P. E., \& David Ruales, C. A. (2015). Reproducción inducida de la sabaleta Brycon henni: revisión bibliográfica. Revista Lasallista de Investigación, 12(1), 211-220.

Magallanes, H. (1989). Evaluación íctica del sistema oriente antioqueno (Tesis de pregrado). Universidad de Antioquia, Medellín, Colombia.

Mancera-Rodríguez, N. J., Castellanos-Barliza, J., \& Urrego-Ballestas, D. (2016) Biología reproductiva de Saccodon dariensis (Teleostei: Parodontidae) 
en afluentes del río Guatapé, cuenca del río Magdalena, Colombia. Revista de Biología Tropical, 64(2), 635-653.

Martínez-Orozco, H. J., \& Vásquez-Zapata, G. L. (2001). Aspectos reproductivos de la sabaleta Brycon henni (Piscis: Characidae) en el embalse de Salvajina, Colombia. Revista de la Asociación Colombiana de Ictiólogos - Dahlia, 4, 75-82.

Montoya-López, A. F., Carrillo, L. M., \& Olivera, M. (2006a). Algunos aspectos biológicos y del manejo en cautiverio de la Sabaleta Brycon henni Eigenmann, 1913 (Pisces: Characidae). Revista Colombiana de Ciencias Pecuarias, 19(2), 180-186.

Montoya-López, A. F., Tabares, C. J., Echeverri, A., Arboleda, L., \& Olivera-Ángel, M. (2006b). Descripción anatómica e histológica de las gónadas en Sabaleta (Brycon henni, Eigenmann 1913). Revista Colombiana de Ciencias Pecuarias, 19(2), 187-196.

Perdomo, J. (1978) La Sabaleta (Brycon henni) observaciones bioecológicas y su importancia como especie de cultivo. Revista Divulgación Pesquera, 11(1), 1-46.

Restrepo-Escobar, N., Hurtado-Alarcón, J. C., ManceraRodríguez, N. J., \& Márquez, E. J. (2016). Variations of body geometry in Brycon henni (Teleostei: Characiformes, Bryconidae) in different rivers and streams. Journal of Fish Biology, 89, 522-528.

Selman, K., \& Wallace, R. A. (1989). Cellular aspects of oocyte growth in teleosts. Zoological Science, $6,211-231$
Sharpe, D., \& Hendry, A. (2009). Life history change in commercially exploited fish stocks: an analysis of trends across studies. Evolutionary Applications, $2,260-275$

Usma, J. S. (2001). Peces de la Cuenca media del río Patía y el río Güiza, Nariño, Colombia. Cespedesia, 24(7578), 7-25.

Vazzoler, A. E.A. de M. (1996). Biologia da reproducao de peixes teleosteos: teoria e pratica: Maringa. Sao Pablo, Brazil: EDUEM.

Wentworth, C. (1922). A scale of grade and class terms for clastic sediments. The Journal of Geology, 30, 377-392.

Winemiller, K. O. (1989). Patterns of variation in life history among South American fishes in seasonal environments. Oecologia, 81, 225-241.

Winemiller, K. O. (1990). Spatial and temporal variation in tropical fish trophic networks. Ecological Monographs, 60, 331-362.

Wootton, R. (1999). Ecology of Teleost Fishes, London, England: Chapman \& Hall.

Zuluaga-Gómez, A., Giarrizzo, T., Andrade, M., \& ArangoRojas, A. (2014). Length-weight relationships of 33 selected fish species from the Cauca River Basin, trans-Andean region, Colombia. Journal of Applied Ichthyology, 30, 1077-1080. 